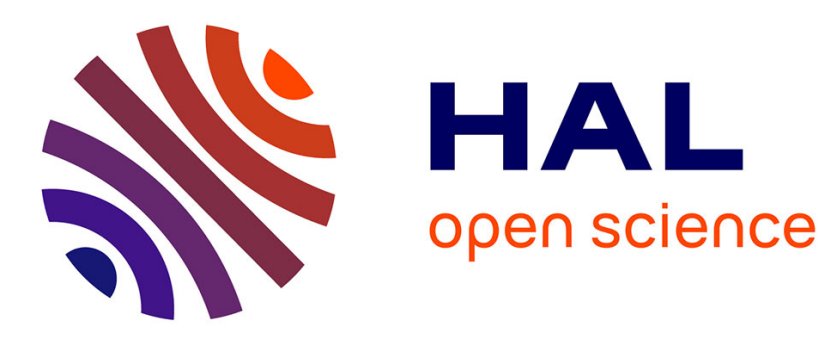

\title{
Plunge milling time optimization via mixed-integer nonlinear programming
}

Sonia Cafieri, Frederic Monies, Marcel Mongeau, Christian Bes

\section{To cite this version:}

Sonia Cafieri, Frederic Monies, Marcel Mongeau, Christian Bes. Plunge milling time optimization via mixed-integer nonlinear programming. Computers \& Industrial Engineering, 2016, 98, pp.434-445. 10.1016/j.cie.2016.06.015 . hal-01337391

\section{HAL Id: hal-01337391 \\ https://hal-enac.archives-ouvertes.fr/hal-01337391}

Submitted on 26 Aug 2016

HAL is a multi-disciplinary open access archive for the deposit and dissemination of scientific research documents, whether they are published or not. The documents may come from teaching and research institutions in France or abroad, or from public or private research centers.
L'archive ouverte pluridisciplinaire HAL, est destinée au dépôt et à la diffusion de documents scientifiques de niveau recherche, publiés ou non, émanant des établissements d'enseignement et de recherche français ou étrangers, des laboratoires publics ou privés. 


\title{
Plunge milling time optimization via mixed-integer nonlinear programming
}

\author{
Sonia Cafieri ${ }^{1}$, Frédéric Monies ${ }^{2}$, Marcel Mongeau ${ }^{1}$, and \\ Christian Bes ${ }^{2}$ \\ ${ }^{1}$ ENAC, MAIAA, F-31055 Toulouse, France, Université de \\ Toulouse, IMT, F-31400 Toulouse, France \\ ${ }^{2}$ Université de Toulouse 3, ICA (Institut Clément Ader), \\ F-31062 Toulouse, France
}

\begin{abstract}
Plunge milling is a recent and efficient production mean for machining deep workpieces, notably in aeronautics. This paper focuses on the minimization of the machining time by optimizing the values of the cutting parameters. Currently, neither Computer-Aided Manufacturing (CAM) software nor standard approaches take into account the tool path geometry and the control laws driving the tool displacements to propose optimal cutting parameter values, despite their significant impact. This paper contributes to plunge milling optimization through a Mixed-Integer NonLinear Programming (MINLP) approach, which enables us to determine optimal cutting parameter values that evolve along the tool path. It involves both continuous (cutting speed, feed per tooth) and, in contrast with standard approaches, integer (number of plunges) optimization variables, as well as nonlinear constraints. These constraints are related to the Computer Numerical Control (CNC) machine tool and to the cutting tool, taking into account the control laws. Computational results, validated on CNC machines and on representative test cases of engine housing, show that our methodology outperforms standard industrial engineering know-how approaches by up to $55 \%$ in terms of machining time.
\end{abstract}




\section{Introduction}

Several industrial processes, arising for instance in the aeronautical industry, are based on material removing (milling) that can be performed by several techniques. For aeronautical workpieces, the quantity of material to be removed often represents a very large proportion of the stock material. The most efficient milling processes include: high-speed machining [18, 7], inclined milling with balancing of the transverse cutting forces $[9,15]$, and plunge millling $[16,6,5]$. Among them, plunge milling is a recognized highly-efficient process thanks to its high removal rate due to its distribution of cutting forces on the tool. More precisely, the radial force that causes chatter is reduced and the axial cutting force generally compresses the tool on the spindle and then increases its stiffness. This strategy is less subject to vibration than the other above-mentioned milling techniques. This is especially crucial for deep milled workpieces. Plunge milling is essentially used for making vertical walls (lateral plunge milling), enlarging holes, or slotting [23]. In the case of lateral plunge milling, the tool moves parallel to the wall to be produced [22]. The thickness of the wall to be milled determines the radial depth of cut. When plunge milling is used to enlarge holes, all the teeth of the tool cut simultaneously, and the radial depth of cut corresponds to the difference between the radius of the pre-existing hole and that of the tool. In the case of slotting (also referred to as full-slot plunge milling), the tool is fully engaged into the material to be milled (see Fig. 1), the cutting width being equal to the tool diameter [5]. Plunge milling is also called z-axis milling, in three-axis machining. It is composed of a sequence of cycles which are repeated along a guide curve provided by a Computer-Aided Manufacturing (CAM) software. Each cycle includes three phases: plunging, rising, and offset [16]. The tool removes material during the plunging phase in the z-axis. Then, it retracts during the rising phase. Finally, it steps over in the $\mathrm{x}$ - and/or $\mathrm{y}$-axis during the offset phase so as to make an overlapping vertical cut at the next cycle (Fig. 1).

Recent research on plunge milling optimization focuses on geometry tool selection, tool path generation, cutting parameters, and kinematic capabilities of the machine tools. According to the type of operation (example: roughing pockets, roughing turbine blades,...), the tool path can be optimized. For example, Ren et al. [17] and Sun et al. [20] study plunge milling tool-path generation. The former optimize the machining time, while the latter concentrate on improving the cutting efficiency and increasing the life 


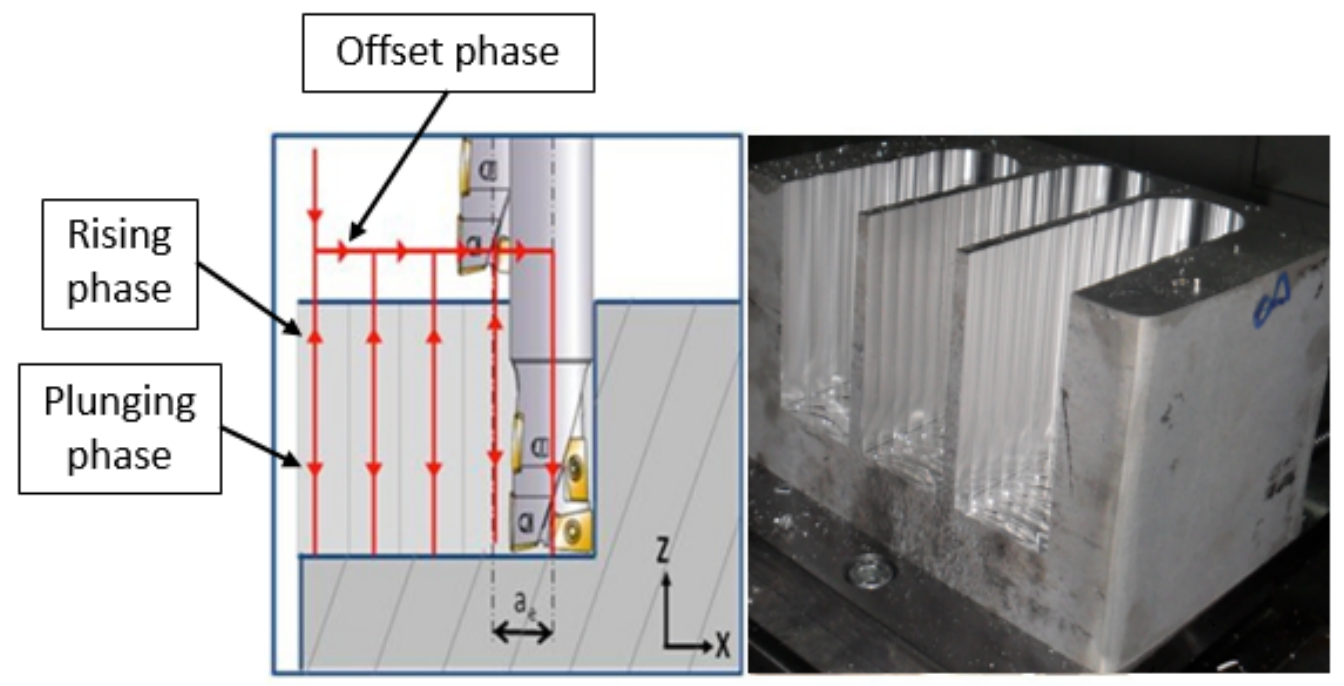

Figure 1: Plunge Milling

time. More recently, Han et al. [10] propose a method for optimizing both the plunge tool selection and the tool path generation in the case of rough machining of free-form surface impellers. Remark that in the context of pocket milling, [1] also optimizes both the tool-path generation and the machine-tool speed (feed) under cutting-force constraints.

Studies that rely on the cutting parameter optimization to improve the plunge milling efficiency are relatively rare. These parameters determine the cutting forces, the power consumption of the spindle, the stability in machining, and the metal removal rate. Zhuang et al. [22] propose an optimization of some cutting parameters in the case of lateral plunge milling. They consider constraints on cutting forces, cutting parameters, and stability criteria and use the frequency domain method defined in [12]. Their objective is to maximize the metal removal rate by optimizing the radial depth of cut, the radial offset and the cutting speed. However, in their study, the feed per tooth is not optimized, its value being kept fixed during the optimization process. Furthermore, this optimization is performed by a simple heuristic approach, yielding sub-optimal solutions.

Important factors in machining time include the kinematic capabilities (jerk, acceleration, and maximum speed) of the Computer Numerical Control (CNC) machine tool and the associated control laws. Rauch \& Hascoet 
present in [16] the impact of these factors, and also an analysis of the performances of plunge milling as a function of both the machine-tool kinematics capabilities and of one specific control law. Despite the relevance of these factors in machining performances, none of the above approaches optimizes simultaneously all the major cutting parameters under kinematic constraints. Moreover, to the authors' knowledge, no work on cutting parameter optimization takes into account the lengths of the tool trajectories.

In this paper, we propose a new methodology for optimizing the cutting parameters in order to minimize the plunge milling time. We consider the case of full-slot plunge milling, to mill deep workpieces (casings), like aeronautical pockets. This is the most critical case in terms of cutting forces acting on the tool, as this is the situation where the cutting forces reach their maximum values [5]. Furthermore, since the cutting width (and therefore, the radial depth of cut) is equal to the tool diameter, one can see that in fullslot plunge milling the main cutting parameters to optimize are the cutting speed, the feed per tooth, and the radial offset. Note that the methodology proposed in the present work can straightforwardly be extended to lateral plunge milling, simply by taking into account the radial depth of cut instead of the tool diameter to determine the maximum cutting forces acting on the tool.

In the present study, machining capabilities, geometry tool selection (diameter, length and number of teeth), and tool path trajectories are supposed to be given (either from manufacturers, from the previously-mentioned methods, or from commercial software).

The optimization methodology to optimize cutting parameters that we propose has the following key novelties. It is based on mixed-integer nonlinear programming, where we optimize, among the cutting parameters, the number of plunges, treated as an integer variable. This is in contrast with considering, as it is usually done, the radial offset, that is a real number (and should then be associated to a continuous variable). Thanks to the optimization of the (integer) number of plunges, we are able to decompose the problem into a sequence of optimization subproblems, each of which corresponds to an elementary tool trajectory. Standard approaches consider a real-number value for the radial offset that is common to all elementary trajectories, yielding thereby suboptimal solutions (cutting parameters found are independent of the length of the tool trajectories). This is in contrast to our approach, where the length of each elementary trajectory is taken into account. Hence, we can determine specific values of the cutting parameters, 
tailored for each elementary trajectory. Another strength of the methodology introduced in this paper is the fact that control laws are taken into account in both time-machining and kinematic constraints in the plunge milling process. Indeed control laws have a significant impact on the optimal cutting parameter values.

We propose a mathematical formulation of the problem based on MixedInteger NonLinear Programming (MINLP) [2]. This permits to take into account both the continuous optimization variables (cutting speed, feed per tooth) and the integer optimization variables (number of plunges). Moreover, nonlinear constraints due to both the CNC machine tool and to the tool characteristics are directly taken into account by this formulation. They include constraints related to: the maximum acceleration, the maximum jerk ability, the maximum speed along each axis during the three different phases of a plunge-milling cycle, the control law behaviour, the maximum power machining, the maximum cutting forces, and the cutting parameter bounds. The objective function of the MINLP optimization problem is the machining time, to be minimized. It is the sum of the total (over all cycles) plunging time, rising time, and offset time. These times are function of the given trajectories, the cutting parameters and, of course, of the previously-mentioned constraints. Once the formulation of the MINLP model is established, efficient MINLP solvers can be used to get optimal cutting parameters. The usefulness of this approach for industrial applications is demonstrated on representative test cases of engine housing.

This paper is organized as follows. Section 2 describes the plunge milling context and surveys the different factors that drive the process. These are related to the tool, the material, the workpiece and the machine-tool. Using these driving factors, Section 3 presents one of our main contributions: a formulation of the plunge milling time optimization problem via MINLP. In Section 4, we first propose the detailed analytical expressions of the objective and the constraint functions that define the plunge milling time minimization problem. Then, we report and discuss the results of numerical experiments on representative engine housing test cases. Finally, Section 5 draws conclusions. 


\begin{tabular}{|c|c|}
\hline \multicolumn{2}{|c|}{ Nomenclature } \\
\hline \multicolumn{2}{|c|}{ - Decision variables: } \\
\hline$V_{c}$ & cutting speed $(\mathrm{m} / \mathrm{min})$ \\
\hline$f_{z}$ & feed per tooth $(\mathrm{mm} / \mathrm{rev} /$ tooth $)$ \\
\hline$a_{e}$ & radial offset $(\mathrm{mm})$ \\
\hline$N_{p}$ & number of plunges \\
\hline \multicolumn{2}{|c|}{ - Input parameters: } \\
\hline$L$ & elementary trajectory length $(\mathrm{mm})$ \\
\hline$L_{p}$ & plunging length (mm) \\
\hline$L_{r}$ & rising length $(\mathrm{mm})$ \\
\hline$L_{o}$ & offset length (mm) \\
\hline$T$ & machining time $(\mathrm{s})$ \\
\hline$t_{p}$ & plunging time $(\mathrm{s})$ \\
\hline$t_{r}$ & rising time $(\mathrm{s})$ \\
\hline$t_{o}$ & offset time $(\mathrm{s})$ \\
\hline$i$ & label of an axis $(i \in\{x, y, z\})$ \\
\hline$V_{f}$ & programmed feedrate $(\mathrm{m} / \mathrm{min})$ \\
\hline$A(t)$ & acceleration vector at time $t\left(\mathrm{~m} . \mathrm{s}^{-2}\right)$ \\
\hline$A^{\max }$ & maximum absolute value of $A(t)\left(\mathrm{m}_{\mathrm{s}} \mathrm{s}^{-2}\right)$ \\
\hline$V_{s}(t)$ & speed vector at time $t(\mathrm{~m} / \mathrm{min})$ for the Soft control law \\
\hline$V_{b}(t)$ & speed vector at time $t(\mathrm{~m} / \mathrm{min})$ for the Brisk control law \\
\hline$V_{s}^{\max }$ & maximum value of $V_{s}(t)(\mathrm{m} / \mathrm{min})$ \\
\hline$V_{b}^{\max }$ & maximum value of $V_{b}(t)(\mathrm{m} / \mathrm{min})$ \\
\hline$Z$ & number of teeth \\
\hline$D$ & tool diameter $(\mathrm{mm})$ \\
\hline$P^{\max }$ & maximum machining power $(\mathrm{kW})$ \\
\hline$F_{t}^{\max }$ & maximum tangential cutting force $(\mathrm{N})$ \\
\hline$F_{r}^{\max }$ & maximum radial cutting force $(\mathrm{N})$ \\
\hline$F_{a}^{\text {max }}$ & maximum axial cutting force $(\mathrm{N})$ \\
\hline \multicolumn{2}{|c|}{ - Lower and upper bounds: } \\
\hline$P^{M}$ & maximum machining power upper bound $(\mathrm{kW})$ \\
\hline$F_{t}^{M}$ & maximum tangential cutting force upper bound $(\mathrm{N})$ \\
\hline$F_{r}^{M}$ & maximum radial cutting force upper bound $(\mathrm{N})$ \\
\hline$F_{a}^{M}$ & maximum axial cutting force upper bound $(\mathrm{N})$ \\
\hline $\begin{array}{l}a \\
V_{f}^{M}\end{array}$ & maximum axis speed reachable $(\mathrm{m} / \mathrm{min})$ \\
\hline $\begin{array}{l}f \\
V_{R}^{M}\end{array}$ & maximum rapid speed $(\mathrm{m} / \mathrm{min})$ \\
\hline$A^{M}$ & maximum axis acceleration reachable $\left(\mathrm{m} . \mathrm{s}^{-2}\right)$ \\
\hline$J^{M}$ & maximum axis jerk $\left(\mathrm{m} \cdot \mathrm{s}^{-3}\right)$ \\
\hline \multicolumn{2}{|l|}{$a_{\rho}^{m}, a_{\rho}^{M}$} \\
\hline $\begin{array}{l}f_{z}^{m}, f_{z}^{M} \\
V^{m}, V^{M}\end{array}$ & lower and upper bounds on the decision variables \\
\hline
\end{tabular}




\section{$2 \quad$ Plunge milling process}

In this section, for given tool path trajectories, we overview the different factors that drive the plunge milling process. These are related to the tool, the material, the workpiece and the machine-tool.

More precisely, we describe the cutting parameters, the different phases of a plunge cycle, the machine-tool kinematic characteristics, the control laws providing the temporal evolution profiles of speed, acceleration and jerk, and the cutting forces that act on the tool as well as determine the machining power. These elements will be used in the next section to formalize the optimization problem of minimizing the plunge milling time.

\subsection{Cutting parameters}

The cutting parameters that influence plunge milling are the cutting speed $V_{c}$, the feed per tooth $f_{z}$, and the radial offset $a_{e}$. The radial offset $a_{e}$ (Fig. 1) represents the distance between two successive plunges into the stock and is therefore directly related to the number of plunges. The cutting parameters $V_{c}$ and $f_{z}$ together with the tool parameters (the number of teeth $Z$ and the tool diameter $D$ ) drive the programmed feedrate $V_{f}$, which is given by:

$$
V_{f}=\frac{10^{3} V_{c} f_{z} Z}{\pi D}
$$

The cutting parameters $V_{c}, f_{z}$ and $a_{e}$, influence the cutting forces during the machining. Among these three parameters, $f_{z}$ and $a_{e}$ mainly influence the tool loading, and have the strongest impact on the preponderant cutting forces [5].

\subsection{Plunge milling phases}

The plunge milling process involves performing successive plunge cycles into the material. The three different phases of a cycle are the following (see Fig. 1). The plunging phase is the phase during which the tool removes material while going down. It is performed with a targeted machining speed, or programmed feedrate, noted $V_{f}$ (depends on the cutting parameters). During the rising phase the tool goes up. This phase is carried out in rapid 
motion. Finally, during the offset phase the tool moves (above the workpiece), along the trajectory, so as to make an overlapping cut at the next cycle. This phase is also carried out in rapid motion. All of these three phases are performed at different lengths $\left(L_{p}, L_{r}, L_{o}\right.$, illustrated on Fig. 2).

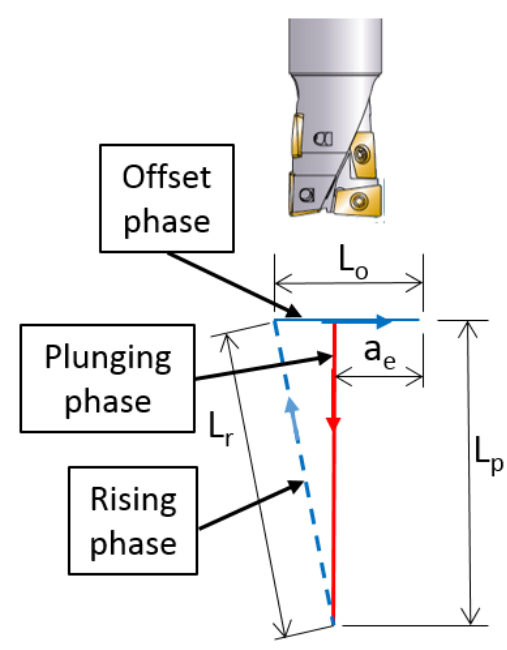

Figure 2: Lengths, $L_{p}, L_{r}, L_{o}$, of the different phases of a plunge-milling cycle

\subsection{Kinematic characteristics}

The machine-tool capabilities to reach the targeted speed and the rapid speed depend both on the machine kinematic characteristics and on the control laws.

The kinematic characteristics are specific to a selected machine and give constraints on speed, acceleration and jerk. They are characterized by the following values:

- $V_{R i}^{M}$, the upper bound on the maximum reachable rapid speed on the $i$-axis $(i \in\{x, y, z\})$ during the rising and offset phases

- $V_{f i}^{M}$, the upper bound on the maximum reachable programmed feedrate on the $i$-axis during the plunging phase

- $A_{i}^{M}$, the upper bound on the $i$-axis for the acceleration $A(t)$ of the machine at time $t$ 
- $J_{i}^{M}$, the upper bound on the $i$-axis jerk of the machine

\subsection{Control laws}

The control laws provide the temporal evolution profiles of speed, acceleration, and jerk of each axis $i$ of the machine, subject to the above upper bounds (kinematic constraints). The programmed feedrate and the rapid motion speed may be reached more or less rapidly following the control law temporal profiles. Although our optimization methodology can be applied in the context of any type of control laws, the tests presented in this paper use the jerk, acceleration and velocity profiles of the Siemens 840D CNC controller: they will be named "Brisk" and "Soft" laws in the sequel of the paper (Fig. 3) [19].

For machining accuracy and workpiece quality reasons, the Soft law is used in the plunging phase. Using the Soft law, the axis motions are driven by constant jerk steps (Fig. 3). Then, the acceleration temporal behaviour is deduced. Finally, the Soft law provides the temporal evolution of the speed $V_{s}(t)$ (whose target value is the feedrate $V_{f}$ ).

During the rising and offset phases, the Brisk law is applied (Fig. 2). Using the Brisk law, the axis motions are driven by constant acceleration steps (Fig. 3). Then, the Brisk law provides the temporal evolution of the rapid speed $V_{b}(t)$ (whose target value is $V_{R}^{M}$ ).

The speed profile control laws can be represented by a piecewise polynomial function of time. For the Soft law (plunging phase), the feedrate profile is described by at most 7 phases (see Fig. 3):

$$
V_{s}(t)=\sum_{j=1}^{7} P_{s}^{j}(t)
$$

where

$$
P_{s}^{j}(t)= \begin{cases}a_{j}+b_{j} t+c_{j} t^{2} & \text { if } t_{j-1} \leqslant t \leqslant t_{j} \quad(j=1, . ., 7) \\ 0 & \text { otherwise }\end{cases}
$$

For each axis $i \in\{x, y, z\}$, the coefficients $a_{j}, b_{j}, c_{j}$ are computed from $A_{i}^{M}$, $J_{i}^{M}, L_{p}$ and the programmed feedrate $V_{f}$, which is a function of $V_{c}, f_{z}, D$, and $Z$. Note that $V_{s}(t) \leqslant V_{f} \leqslant V_{f}^{M}$. 

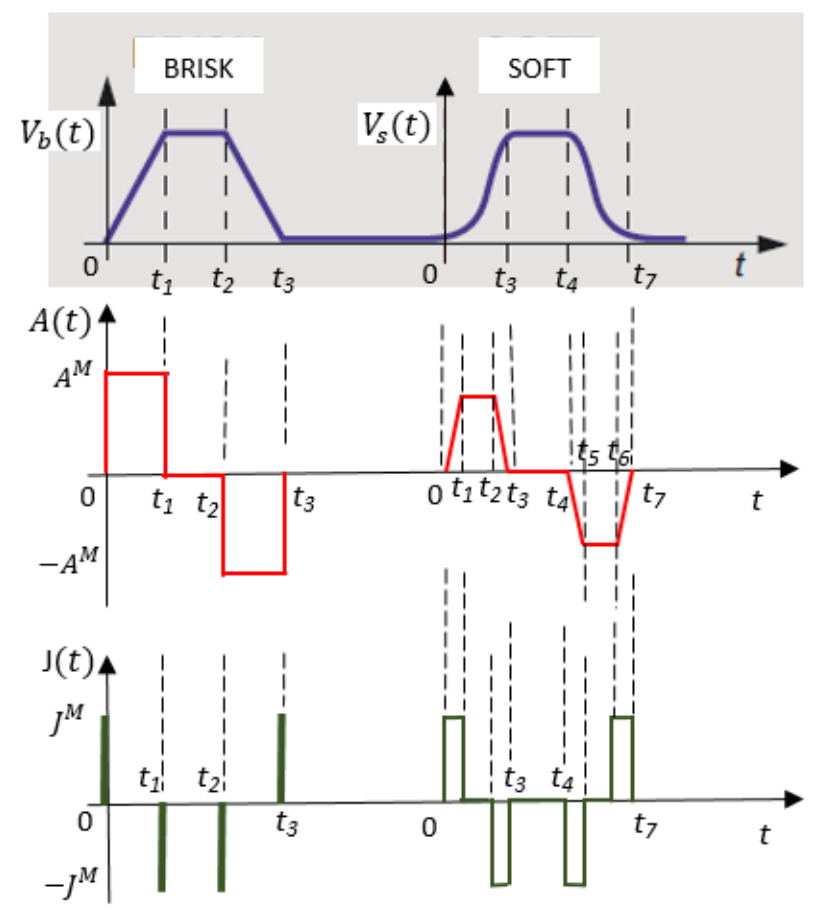

Figure 3: Jerk, acceleration and velocity profiles: Brisk and Soft mode

The different time points $t_{j}, j=1,2, \ldots, 7$, which define the piecewise polynomial function, represent the different jerk phases of the control law following each axis $i$ (see Fig. 3). These time points are functions of the same above parameters. The plunging phase starts at time $t_{0}=0$ and ends at time $t_{7}$. Then, $t_{7}$ is the total plunging time, and it is noted $t_{p}$. Remark that both departure and end times are the same for each axis $i$, we have: $\left(t_{0}\right)_{i}=t_{0}$ and $\left(t_{7}\right)_{i}=t_{7}$. Since plunge milling can be an exact stop check mode [16], it is assumed that the feedrate $V_{s}$ is zero at $t_{0}$ and $t_{7}$. Remark that when $L_{p}$ is small, the number of polynomial pieces can be reduced to five or fewer pieces. For example, phases 2 and 6 (see Fig. 3) may not be present because the maximum acceleration cannot be reached. In Section 4 a precise formulation of the total plunging time $t_{p}$, depending of the number of phases, will be provided, tailored to our case of plunge milling.

The feedrate profile provided by the Brisk law (in the rising and offset phases) is: 


$$
V_{b}(t)=\sum_{j=1}^{3} P_{b}^{j}(t)
$$

where

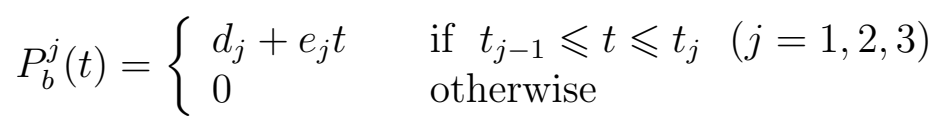

For each axis $i \in\{x, y, z\}$, the coefficients $d_{j}$ and $e_{j}$ are function of $A_{i}^{M}$, $L_{r}\left(\right.$ or $\left.L_{o}\right)$ and of the rapid speed $V_{R i}^{M}$. Note that $V_{b}(t) \leqslant V_{R i}^{M}$.

The different time points $t_{j}$ that define the piecewise linear function represent the different acceleration phases of the control law following each axis. The rising (or offset) phase starts at time $t_{0}=0$, and ends at time $t_{3}$. Remark that $t_{3}$ is equal to $t_{r}$ (respectively, $t_{o}$ ), i.e. the rising (respectively, offset) duration. Both departure and end times are the same for each axis $i$. It is assumed that the feedrate $V_{b}$ is zero at times $t_{0}$ and $t_{3}$. When $L_{r}$ (respectively, $L_{o}$ ) is small, the number of linear pieces is reduced to two pieces. In this case, phase 2 does not exist. In Section 4 a precise formulation of the durations $t_{r}$ and $t_{o}$, depending of the number of phases, will be provided.

The strong impact of control laws on machining time is illustrated in A.

\subsection{Cutting forces and machining power}

Beside the importance of kinematic and control law characteristics for computing accurate time machining, cutting forces have to be evaluated for ensuring tool safe life and to enforce machine power limitation.

The maximum cutting forces acting on the tool are the maximum tangential force $F_{t}^{\max }$, the maximum radial force $F_{r}^{\max }$, and the maximum axial force $F_{a}^{\max }$. Generally, the tangential force is expressed as the product of a specific cutting pressure and the instantaneous chip cross-section [4, 9, 13]. Since the cutting pressure and the chip cross-section are both nonlinearly dependant on the cutting parameters, the maximum tangential force $F_{t}^{\max }$ can be expressed as a nonlinear function of $V_{c}, f_{z}, a_{e}: F_{t}^{\max }\left(V_{c}, f_{z}, a_{e}\right)[4,5,23]$. The radial force is taken proportional to the tangential force [21], just as the axial force. Then, the maximum radial and axial forces are again nonlinear functions of $V_{c}, f_{z}$ and $a_{e}: F_{r}^{\max }\left(V_{c}, f_{z}, a_{e}\right)$ and $F_{a}^{\max }\left(V_{c}, f_{z}, a_{e}\right)$.

Due to both tool limitations (bending, stiffness, vibrations, wear, ...) and maximum loading on machine-tool axis, these forces have to be below critical values: $F_{t}^{\max }\left(f_{z}, V_{c}, a_{e}\right) \leqslant F_{t}^{M}, F_{r}^{\max }\left(f_{z}, V_{c}, a_{e}\right) \leqslant F_{r}^{M}, F_{a}^{\max }\left(f_{z}, V_{c}, a_{e}\right) \leqslant$ 
$F_{a}^{M}$ where $F_{t}^{M}, F_{r}^{M}, F_{a}^{M}$ are respectively the upper bounds on the maximum allowable tangential, radial and axial cutting force. Concerning the maximum machining power, noted $P^{\max }$, it can be approximated by the product of the maximum tangential force $F_{t}^{\max }$ and the cutting speed $V_{c}$, that is: $P^{\max } \simeq$ $F_{t}^{\max } V_{c}$. It has to be below an upper bound value, generally given by the machine tool: $P^{\max } \leqslant P^{M}$.

\section{Optimization formulation}

In this section, we present our contribution on plunge milling optimization through a mixed-integer nonlinear programming formulation. The aim is to propose optimized cutting parameter values that take into account the lengths of the tool trajectories, the machine-tool performances and limitations (kinematic characteristics, control laws, maximum machining power) and the tool characteristics. We consider that the machining capabilities $\left(A^{M}, J^{M}, V_{f}^{M}\right)$, the tool characteristics $(D, Z$, and its length), the length $L_{p}$ of the workpiece, and the tool path trajectory lengths are given. Tool trajectories are defined by successive elementary tool paths such as: straight lines, arc circles and polynomial curves. In this paper, we decompose the optimization of the total machining time into independent optimization subproblems, each of which corresponds to an elementary tool path. Then, the global solution is obtained by assembling the solutions (the cutting parameter values) of these subproblems. This permits to propose specific optimal cutting parameter values tailored to each elementary trajectory. Compared to optimized cutting parameter taking the same values for all elementary trajectories, this leads to a solution that is more time efficient for machining the workpiece. Consequently, in this section we present an optimization formulation for an elementary tool path with a given length, $L$.

We propose a mathematical formulation of the problem at hand under the form of an optimization problem, and more precisely of a mixed-integer nonlinear programming (MINLP) problem. Nonlinearities arise from the underlying physical process. We propose a formulation involving continuous as well as integer optimization variables. The integer variables come from the discrete number of plunges and from modeling piecewise-defined functions due to the control laws that impact the machining time. To address these 
difficulties, we take advantage of recent advances in MINLP [14].

The optimization variables are: the cutting parameters $\left(V_{c}, f_{z}, a_{e}\right)$ and the number of plunges $N_{p}$. The number of plunges, $N_{p}$, is bounded to be integer-valued (integer number of plunges for the given length of an elementary trajectory). Moreover, there is a direct relationship between the number of plunges and the radial offset $a_{e}$ :

$$
N_{p}=L / a_{e}
$$

Therefore, the optimization variables can in fact be reduced to: $V_{c}, f_{z}, N_{p}$ (and $a_{e}$ will be straightforwardly deduced from equation (6) after optimization). The first two variables $\left(V_{c}, f_{z}\right)$ are continuous and the last one, $N_{p}$, is integer. Remark that common practice $[11,22]$ optimizes over the three continuous variables $V_{c}, f_{z}, a_{e}$, resulting in a value of $N_{p}$ that is not integervalued and must be rounded to the next integer. This leads to an extra plunge which is likely to be inefficient. This loss of efficiency is summed up for each elementary trajectory, clearly yielding to a suboptimal solution.

For the sake of simplicity, and because the proposed formulation can easily be extended, we assume that:

- the characteristics of each axis $(x, y, z)$ are the same: we denote, for $i \in\{x, y, z\}, A_{i}^{M}=A^{M}, A_{i}^{\max }=A^{\max }, V_{f i}^{M}=V_{f}^{M}$ and $V_{R i}^{M}=V_{R}^{M}$;

- the length, $L_{r}$, of the rising phases and that, $L_{p}$, of the plunging phases are equal and these phases involve only the z-axis;

- the length, $L_{o}$, of the offset phases is equal to the radial offset $a_{e}$ : $L_{o}=a_{e}$;

- all of these lengths are constant along a given elementary trajectory.

\subsection{Objective function}

The objective function to be minimized is the machining time which can be expressed as:

$$
T\left(V_{c}, f_{z}, N_{p}\right)=N_{p}\left(t_{p}\left(V_{c}, f_{z}\right)+t_{r}+t_{o}\left(N_{p}\right)\right)
$$

where the plunging time, $t_{p}$, the rising time, $t_{r}$, and the offset time, $t_{o}$, functions are described in the next subsections. 


\subsubsection{Plunging time, $t_{p}$}

We define the plunging time as

$$
t_{p}\left(V_{c}, f_{z}\right)=\left\{\begin{array}{lll}
t_{p 1}\left(V_{c}, f_{z}\right), & \text { if } V_{s}^{\max }\left(V_{c}, f_{z}\right)=V_{f}\left(V_{c}, f_{z}\right) \text { and } A^{\max }\left(V_{c}, f_{z}\right)=A^{M} \\
t_{p 2}\left(V_{c}, f_{z}\right), & \text { if } V_{s}^{\max }\left(V_{c}, f_{z}\right)=V_{f}\left(V_{c}, f_{z}\right) \text { and } A^{\max }\left(V_{c}, f_{z}\right)<A^{M} \\
t_{p 3}\left(V_{c}, f_{z}\right), & \text { if } V_{s}^{\max }\left(V_{c}, f_{z}\right)<V_{f}\left(V_{c}, f_{z}\right) \text { and } A^{\max }\left(V_{c}, f_{z}\right)=A^{M} \\
t_{p 4}\left(V_{c}, f_{z}\right), & \text { if } V_{s}^{\max }\left(V_{c}, f_{z}\right)<V_{f}\left(V_{c}, f_{z}\right) \text { and } A^{\max }\left(V_{c}, f_{z}\right)<A^{M},
\end{array}\right.
$$

where:

- $V_{s}^{\max }$ is the maximum speed that can be reached following the Soft control law. It is a function of the given data: $L_{p}, J^{M}, A^{M}, D, Z$, and of the optimization variables $V_{c}, f_{z}$. Obviously, we always have $0 \leqslant V_{s}^{\max }\left(V_{c}, f_{z}\right) \leqslant V_{f}\left(V_{c}, f_{z}\right)$.

- $V_{f}$ is a function of the given data, $D, Z$, and of the optimization variables $V_{c}, f_{z}$.

- $A^{\max }$ is a function of $J^{M}$ and $V_{f}$. Obviously, we always have $0 \leqslant$ $A^{\max }\left(V_{c}, f_{z}\right) \leqslant A^{M}$.

Since $L_{p}, J^{M}, A^{M}, D, Z$ are given data, the above quantities are only function of the two optimization variables: $V_{c}, f_{z}$. The expression of $V_{f}\left(V_{c}, f_{z}\right)$ is given in equation (1). The analytic formulas that enable to compute $t_{p}\left(V_{c}, f_{z}\right)$, $V_{s}^{\max }\left(V_{c}, f_{z}\right)$, and $A^{\max }\left(V_{c}, f_{z}\right)$ will be provided in section 4 because these functions depend on the selected control law.

To summarize,

- $t_{p 1}$ represents the plunging time associated with a displacement length $L_{p}$ when the maximum feedrate $V_{s}^{\max }$ reaches $V_{f}$ and the maximum acceleration $A^{\max }$ reaches $A^{M}$.

- $t_{p 2}$ represents the plunging time associated with a displacement length $L_{p}$ when the maximum feedrate $V_{s}^{\max }$ reaches $V_{f}$ and the maximum acceleration $A^{\text {max }}$ is below $A^{M}$.

- $t_{p 3}$ represents the plunging time associated with a displacement length $L_{p}$ when the maximum feedrate $V_{s}^{\max }$ is below $V_{f}$ and the maximum acceleration $A^{\max }$ reaches $A^{M}$. 
- $t_{p 4}$ represents the plunging time associated with a displacement length $L_{p}$ when the maximum feedrate $V_{s}^{\max }$ is below $V_{f}$ and the maximum acceleration $A^{\max }$ is below $A^{M}$.

\subsubsection{Rising time, $t_{r}$}

The rising time is given by:

$$
t_{r}= \begin{cases}t_{r 1}, & \text { if } V_{b}^{\max }=V_{R}^{M} \\ t_{r 2}, & \text { if } V_{b}^{\max }<V_{R}^{M}\end{cases}
$$

where:

- $t_{r 1}$ represents the rising time associated with a displacement length $L_{r}=L_{p}$, when the (given) rapid speed, $V_{R}^{M}$, is reached.

- $t_{r 2}$ is the rising time when $V_{b}^{\max }$ is below $V_{R}^{M}$. Both $t_{r 1}$ and $t_{r 2}$ are independent of the optimization variables.

- $V_{b}^{\max }$ is the maximum speed that can be reached following the Brisk control law. It is a function of the rising length, $L_{r}$, and of the maximum acceleration reachable, $A^{M}: V_{b}^{\max }=\varphi\left(A^{M}, L_{r}\right)$, where $\varphi$ is a function that is known. Since $L_{r}$ and $A^{M}$ are given data, $V_{b}^{\max }$ does not depend upon the optimization variables, therefore $V_{b}^{\max }=\kappa$, where $\kappa$ is a constant in the optimization problem. Note that $V_{b}^{\max }$ is always below $V_{R}^{M}: 0 \leqslant V_{b}^{\max }=\kappa \leqslant V_{R}^{M}$. The analytic formulas that permit to compute $t_{r}, V_{b}^{\max }$, and thereby $t_{r}$, will be provided in the test cases presented in Section 4.

\subsubsection{Offset time, $t_{o}$}

The offset time, $t_{o}$, could also be defined in a piecewise manner, as for the rising time (equation (9)). However, since the displacement length, $L_{o}=$ $a_{e}=L / N_{p}$, is always sufficiently small due to technological constraints, the condition $V_{b}^{\max }=V_{R}^{M}$ is never met. The analytic formulas that enable to compute $t_{o}$ will be provided in Section 4 . 


\subsection{Constraints}

There are two distinct sets of constraints. First, there are disjunctive constraints that allow to model the piecewise-defined functions that appear in the objective function; second, those that model the physics of the system.

\subsubsection{Disjunctive constraints}

The definition of the objective function involves disjunctions as shown by equations (8) and (9). More precisely, $T$ is a continuous piecewise-defined function of the optimization variables $V_{c}, f_{z}$, and $N_{p}$. Optimization software cannot handle directly problems involving piecewise-defined functions. In order to obtain a proper mathematical programming formulation, i.e. involving only differentiable functions (without any piecewise-defined functions), we introduce the following binary variables:

- $u_{1}, u_{2}, u_{3}$, and $u_{4}$ for the plunging-time function, $t_{p}$

- $v$ for the rising-time function, $t_{r}$

Using these variables, we can rewrite the piecewise-defined equation (9) as:

$$
t_{r}=v t_{r 1}+(1-v) t_{r 2}
$$

provided that the following constraints are taken into account:

$$
\begin{gathered}
v \in\{0,1\} \\
\left\{\begin{array}{l}
0 \leqslant V_{R}^{M}-\kappa \leqslant(1-v) V_{R}^{M} \\
V_{b}^{\text {max }}-V_{R}^{M}<v .
\end{array}\right.
\end{gathered}
$$

Indeed, one can easily verify, by enumerating the two possible values for $v$, that equation (9) is equivalent to equations (10), (11) and (12).

In an analogous manner, one can show that the piecewise-defined equation (8) can be rewritten as

$$
t_{p}\left(V_{c}, f_{z}\right)=u_{1} t_{p 1}\left(V_{c}, f_{z}\right)+u_{2} t_{p 2}\left(V_{c}, f_{z}\right)+u_{3} t_{p 3}\left(V_{c}, f_{z}\right)+u_{4} t_{p 4}\left(V_{c}, f_{z}\right)
$$

provided that the following constraints are taken into account:

$$
u_{1}, u_{2}, u_{3}, u_{4} \in\{0,1\}
$$




$$
\begin{gathered}
\sum_{j=1}^{4} u_{j}=1 \\
\left\{\begin{array}{l}
0 \leqslant V_{f}\left(V_{c}, f_{z}\right)-V_{s}^{\max }\left(V_{c}, f_{z}\right) \leqslant\left(1-u_{1}\right) V_{f}\left(V_{c}, f_{z}\right) \\
0 \leqslant A^{M}-A^{\max }\left(V_{c}, f_{z}\right) \leqslant\left(1-u_{1}\right) A^{M} \\
0 \leqslant V_{f}\left(V_{c}, f_{z}\right)-V_{s}^{\max }\left(V_{c}, f_{z}\right) \leqslant\left(1-u_{2}\right) V_{f}\left(V_{c}, f_{z}\right) \\
A^{\max }\left(V_{c}, f_{z}\right)-A^{M}<\left(1-u_{2}\right) \\
V_{s}^{\max }\left(V_{c}, f_{z}\right)-V_{f}\left(V_{c}, f_{z}\right)<\left(1-u_{3}\right) \\
0 \leqslant A^{M}-A^{\max }\left(V_{c}, f_{z}\right) \leqslant\left(1-u_{3}\right) A^{M} \\
V_{s}^{\max }\left(V_{c}, f_{z}\right)-V_{f}\left(V_{c}, f_{z}\right)<\left(1-u_{4}\right) \\
A^{\max }\left(V_{c}, f_{z}\right)-A^{M}<\left(1-u_{4}\right)
\end{array}\right.
\end{gathered}
$$

This can easily be verified by enumerating the four possible values for the vector $\left(u_{1}, u_{2}, u_{3}, u_{4}\right):(1,0,0,0),(0,1,0,0),(0,0,1,0)$ and $(0,0,0,1)$.

Finally, using equations (10) and (13), the objective function (7) becomes:

$T\left(V_{c}, f_{z}, N_{p}\right)=N_{p}\left(u_{1} t_{p 1}\left(V_{c}, f_{z}\right)+u_{2} t_{p 2}\left(V_{c}, f_{z}\right)+u_{3} t_{p 3}\left(V_{c}, f_{z}\right)+u_{4} t_{p 4}\left(V_{c}, f_{z}\right)+\right.$

$$
\left.v t_{r 1}+(1-v) t_{r 2}+t_{o}\left(N_{p}\right)\right) \text {. }
$$

Remark that constraints (12) and (15) involve strict inequalities, which cannot be handled by mathematical programming solvers. Therefore, we change strict into large inequalities after subtracting a sufficiently small (userdefined) constant $\epsilon$ from the right-hand-sides:

$$
\left\{\begin{array}{l}
0 \leqslant V_{f}\left(V_{c}, f_{z}\right)-V_{s}^{\max }\left(V_{c}, f_{z}\right) \leqslant\left(1-u_{1}\right) V_{f}\left(V_{c}, f_{z}\right) \\
0 \leqslant A^{M}-A^{\max }\left(V_{c}, f_{z}\right) \leqslant\left(1-u_{1}\right) A^{M} \\
0 \leqslant V_{f}\left(V_{c}, f_{z}\right)-V_{s}^{\max }\left(V_{c}, f_{z}\right) \leqslant\left(1-u_{2}\right) V_{f}\left(V_{c}, f_{z}\right) \\
A^{\max }\left(V_{c}, f_{z}\right)-A^{M} \leq\left(1-u_{2}\right)-\epsilon \\
V_{s}^{\max }\left(V_{c}, f_{z}\right)-V_{f}\left(V_{c}, f_{z}\right) \leq\left(1-u_{3}\right)-\epsilon \\
0 \leqslant A^{M}-A^{\max }\left(V_{c}, f_{z}\right) \leqslant\left(1-u_{3}\right) A^{M} \\
V_{s}^{\max }\left(V_{c}, f_{z}\right)-V_{f}\left(V_{c}, f_{z}\right) \leq\left(1-u_{4}\right)-\epsilon \\
A^{\max }\left(V_{c}, f_{z}\right)-A^{M} \leq\left(1-u_{4}\right)-\epsilon
\end{array}\right.
$$

and

$$
\left\{\begin{array}{l}
0 \leqslant V_{R}^{M}-\kappa \leqslant(1-v) V_{R}^{M} \\
V_{b}^{\max }-V_{R}^{M} \leq v-\epsilon
\end{array}\right.
$$




\subsubsection{Cutting parameter bounds}

Due to material and tool characteristics, cutting parameters have to satisfy the following lower-bound and upper-bound inequalities:

$$
\begin{gathered}
V_{c}^{m} \leqslant V_{c} \leqslant V_{c}^{M} \\
f_{z}^{m} \leqslant f_{z} \leqslant f_{z}^{M} \\
a_{e}^{m} \leqslant a_{e} \leqslant a_{e}^{M}
\end{gathered}
$$

where $V_{c}^{m}, V_{c}^{M}, f_{z}^{m}, f_{z}^{M}, a_{e}^{m}, a_{e}^{M}$ are given data (depending upon the material and the machine features). Remark that the last equation is equivalent to

$$
\frac{L}{a_{e}^{M}} \leq N_{p} \leq \frac{L}{a_{e}^{m}}
$$

since $N_{p}=\frac{L}{a_{e}}$.

\subsubsection{Machine kinematics}

The kinematic characteristics of the machine tool imply the following inequalities:

- The programmed feedrate, $V_{f}$, has to be smaller than $V_{f}^{M}: V_{f}\left(V_{c}, f_{z}\right) \leqslant$ $V_{f}^{M}$.

- The maximum acceleration, $A^{\max }$, has to be smaller than $A^{M}: A^{\max }\left(V_{c}, f_{z}\right) \leqslant$ $A^{M}$.

\subsubsection{Cutting forces}

The constraints due to cutting forces are defined as follows:

- The maximum tangential cutting force $F_{t}^{\max }\left(V_{c}, f_{z}, N_{p}\right)$ has to be smaller than a maximum cutting force which is a given value of the tool characteristics. Therefore, this constraint can be written as $F_{t}^{\max }\left(V_{c}, f_{z}, N_{p}\right) \leqslant$ $F_{t}^{M}$. In the test cases presented in the next section, the chosen model for $F_{t}^{\max }$ will be specified.

- The maximum radial cutting force $F_{r}^{\max }\left(V_{c}, f_{z}, N_{p}\right)$ has to be smaller than a maximum cutting force which is a given value of the tool characteristics: $F_{r}^{\max }\left(V_{c}, f_{z}, N_{p}\right) \leqslant F_{r}^{M}$. 
- The maximum axial cutting force $F_{a}^{\max }\left(V_{c}, f_{z}, N_{p}\right)$ has to be smaller than a maximum cutting force which is a given value of the tool and the machine-tool characteristics: $F_{a}^{\max }\left(V_{c}, f_{z}, N_{p}\right) \leqslant F_{a}^{M}$.

- The maximum power associated with cutting can be approximated by $P^{\max } \simeq F_{t}^{\max }\left(V_{c}, f_{z}, N_{p}\right) V_{c}$. The maximum power has to be smaller than the given maximum machining power $P^{M}$. Therefore this constraint can be written as $P^{\max }\left(V_{c}, f_{z}, N_{p}\right) \leqslant P^{M}$.

\subsubsection{MINLP formulation}

To summarize, the minimization of time machining can be formalized as the following MINLP optimization problem:

$$
\begin{aligned}
\underset{u, v, V_{c}, f_{z}, N_{p}}{\operatorname{minimize}} T\left(V_{c}, f_{z}, N_{p}\right) & =N_{p}\left(u_{1} t_{p 1}\left(V_{c}, f_{z}\right)+u_{2} t_{p 2}\left(V_{c}, f_{z}\right)\right. \\
& +u_{3} t_{p 3}\left(V_{c}, f_{z}\right)+u_{4} t_{p 4}\left(V_{c}, f_{z}\right) \\
& \left.+v t_{r 1}+(1-v) t_{r 2}+t_{o}\left(N_{p}\right)\right)
\end{aligned}
$$

subject to:

$$
\begin{gathered}
u_{j} \in\{0,1\} \quad 1 \leqslant j \leqslant 4, \quad \sum_{j=1}^{4} u_{j}=1 \\
v \in\{0,1\}
\end{gathered}
$$

constraints (16) and (17)

Cutting parameters constraints:

$$
\left\{\begin{array}{l}
V_{c}^{m} \leqslant V_{c} \leqslant V_{c}^{M} \\
f_{z}^{m} \leqslant f_{z} \leqslant f_{z}^{M} \\
\frac{L}{a_{e}^{M}} \leqslant N_{p} \leqslant \frac{L}{a_{e}^{m}}
\end{array}\right.
$$


Kinematic constraints:

$$
\begin{aligned}
V_{f}\left(V_{c}, f_{z}\right) & \leqslant V_{f}^{M} \\
A^{\max }\left(V_{c}, f_{z}\right) & \leqslant A^{M}
\end{aligned}
$$

Cutting-force constraints:

$$
\begin{aligned}
F_{t}^{\max }\left(V_{c}, f_{z}, N_{p}\right) & \leqslant F_{t}^{M} \\
F_{r}^{\max }\left(V_{c}, f_{z}, N_{p}\right) & \leqslant F_{r}^{M} \\
F_{a}^{\max }\left(V_{c}, f_{z}, N_{p}\right) & \leqslant F_{a}^{M} \\
F_{t}^{\max }\left(V_{c}, f_{z}, N_{p}\right) V_{c} & \leqslant P^{M}
\end{aligned}
$$

\section{Test cases \& Results}

In this section, we first derive the analytical expressions of the plunging, rising, and offset times, as well as the analytical expressions of constraint functions due to the above-described kinematic characteristics and control laws. We then introduce a cutting-force model that allows us to express the maximum cutting forces and the associated machine power constraints. In the second subsection, we present numerical results obtained when solving the proposed MINLP optimization problem. Comparisons with physical experiments performed on real-life machine tools are provided. These experiments are representative of real milled engine housings.

\subsection{Analytical expressions of the objective and the constraint functions}

In order to solve our problem using efficient optimization methods, we need to provide explicit expressions of the objective and the constraint functions in terms of the three optimization variables $\left(V_{c}, f_{z}\right.$ and $\left.N_{p}\right)$ and of the input data.

\subsubsection{Objective function}

For each plunging cycle, the plunging, rising and offset times are derived by the jerk, acceleration and speed profiles given by the control laws (see Fig. 3). 
We detail next the times involved in the objective function (22).

\section{Plunging time}

Recall that $t_{p}\left(V_{c}, f_{z}\right)$ was given by equation (8). In the test cases considered in this paper, the workpieces are high enough to have the feedrate, $V_{f}$, always attained by $V_{s}^{\max }$, i.e. $V_{s}^{\max }\left(V_{c}, f_{z}\right)=V_{f}\left(V_{c}, f_{z}\right)$. Therefore, only two cases in equation (8) have to be considered: the ones corresponding to $t_{p 1}\left(V_{c}, f_{z}\right)$ and $t_{p 2}\left(V_{c}, f_{z}\right)$. Then, equation (13) becomes:

$$
t_{p}\left(V_{c}, f_{z}\right)=u_{1} t_{p 1}\left(V_{c}, f_{z}\right)+u_{2} t_{p 2}\left(V_{c}, f_{z}\right)
$$

and in the sequel $u_{3}=u_{4}=0$.

Using the Soft law, we have:

- If $A^{\max }\left(V_{c}, f_{z}\right)=A^{M}$, i.e., $u_{1}=1$ and $u_{2}=0$ (which corresponds physically to $\left.V_{f}\left(V_{c}, f_{z}\right) \geq \frac{\left(A^{M}\right)^{2}}{J^{M}}\right)$, then

$$
\begin{aligned}
t_{p}\left(V_{c}, f_{z}\right)=t_{p 1}\left(V_{c}, f_{z}\right) & =2\left(\frac{A^{M}}{J^{M}}+\frac{V_{f}\left(V_{c}, f_{z}\right)}{A^{M}}\right) \\
& +\frac{1}{V_{f}\left(V_{c}, f_{z}\right)}\left(L_{p}-2 Z_{3}\left(V_{c}, f_{z}\right)\right)
\end{aligned}
$$

where:

$V_{f}\left(V_{c}, f_{z}\right)$ is given by equation (1) and:

$$
\begin{aligned}
Z_{3}\left(V_{c}, f_{z}\right) & =Z_{2}\left(V_{c}, f_{z}\right)+V_{2}\left(V_{c}, f_{z}\right)\left(\frac{A^{M}}{J^{M}}\right)+\frac{1}{2} A^{M}\left(\frac{A^{M}}{J^{M}}\right)^{2} \\
& -\frac{1}{6} J^{M}\left(\frac{A^{M}}{J^{M}}\right)^{3} \\
Z_{2}\left(V_{c}, f_{z}\right) & =\frac{1}{6} A^{M}\left(\frac{A^{M}}{J^{M}}\right)^{3}+\frac{1}{2} \frac{\left(A^{M}\right)^{2}}{J^{M}}\left(\frac{V_{f}\left(V_{c}, f_{z}\right)}{A^{M}}-\frac{A^{M}}{J^{M}}\right) \\
& +\frac{1}{2} A^{M}\left(\frac{V_{f}\left(V_{c}, f_{z}\right)}{A^{M}}-\frac{A^{M}}{J^{M}}\right)^{2} \\
V_{2}\left(V_{c}, f_{z}\right) & =\frac{1}{2} \frac{\left(A^{M}\right)^{2}}{J^{M}}+A^{M}\left(\frac{V_{f}\left(V_{c}, f_{z}\right)}{A^{M}}-\frac{A^{M}}{J^{M}}\right)
\end{aligned}
$$


- If $A^{\max }\left(V_{c}, f_{z}\right)<A^{M}$, i.e., $u_{1}=0$ and $u_{2}=1$ (which corresponds physically to $\left.V_{f}\left(V_{c}, f_{z}\right)<\frac{\left(A^{M}\right)^{2}}{J^{M}}\right)$, then

$$
t_{p}\left(V_{c}, f_{z}\right)=t_{p 2}\left(V_{c}, f_{z}\right)=2\left(\frac{A^{\max }\left(V_{c}, f_{z}\right)}{J^{M}}+\frac{V_{f}\left(V_{c}, f_{z}\right)}{A^{\max }\left(V_{c}, f_{z}\right)}\right)+\frac{L_{p}-2 Z_{3}\left(V_{c}, f_{z}\right)}{V_{f}\left(V_{c}, f_{z}\right)}
$$

where:

$$
\begin{aligned}
A^{\max }\left(V_{c}, f_{z}\right)= & \sqrt{J^{M} V_{f}\left(V_{c}, f_{z}\right)} \\
Z_{3}\left(V_{c}, f_{z}\right)= & Z_{2}\left(V_{c}, f_{z}\right)+V_{2}\left(V_{c}, f_{z}\right)\left(\frac{A^{\max }\left(V_{c}, f_{z}\right)}{J^{M}}\right) \\
& +\frac{1}{2} A^{\max }\left(V_{c}, f_{z}\right)\left(\frac{A^{\max }\left(V_{c}, f_{z}\right)}{J^{M}}\right)^{2} \\
& -\frac{1}{6} J^{M}\left(\frac{A^{\max }\left(V_{c}, f_{z}\right)}{J^{M}}\right)^{3} \\
Z_{2}\left(V_{c}, f_{z}\right)= & \frac{1}{6} A^{\max }\left(V_{c}, f_{z}\right)\left(\frac{A^{\max }\left(V_{c}, f_{z}\right)}{J^{M}}\right)^{3} \\
& +\frac{1}{2}\left(\frac{\left(A^{\max }\left(V_{c}, f_{z}\right)\right)^{2}}{J^{M}}\right)\left(\frac{V_{f}\left(V_{c}, f_{z}\right)}{A^{\max }\left(V_{c}, f_{z}\right)}-\frac{A^{\max }\left(V_{c}, f_{z}\right)}{J^{M}}\right) \\
& +\frac{1}{2} A^{\max }\left(V_{c}, f_{z}\right)\left(\frac{V_{f}\left(V_{c}, f_{z}\right)}{A^{\max }\left(V_{c}, f_{z}\right)}-\frac{A^{\max }\left(V_{c}, f_{z}\right)}{J^{M}}\right)^{2} \\
V_{2}\left(V_{c}, f_{z}\right)= & \frac{1}{2}\left(\frac{\left(A^{\max }\left(V_{c}, f_{z}\right)\right)^{2}}{J^{M}}\right) \\
+ & A^{\max }\left(V_{c}, f_{z}\right)\left(\frac{V_{f}\left(V_{c}, f_{z}\right)}{A^{\max }\left(V_{c}, f_{z}\right)}-\frac{A^{\max }\left(V_{c}, f_{z}\right)}{J^{M}}\right) .
\end{aligned}
$$

\section{Rising time}

Recall that $t_{r}$ was given by equation (9). In most CNC machine tools actually used in industry, the rapid speed $V_{R}^{M}$ is so high that in practice it can rarely be attained. Therefore, only one case has to be considered: the one corresponding to $t_{r 2}$. Then equation (10) becomes:

$$
t_{r}=t_{r 2}
$$

and $v=0$ in our tests. Using the Brisk law, we get:

$$
t_{r}=t_{r 2}=2 \sqrt{\frac{L_{r}}{A^{M}}} .
$$




\section{Offset time}

Recall that (see subsection 3.1.3) the rapid speed $V_{R}^{M}$ cannot be reached during the offset phase. Since the Brisk law is applied again, we obtain the same formula as for the rising phase, by replacing the length $L_{r}$ with $\frac{L}{N_{p}}$ :

$$
t_{o}\left(N_{p}\right)=2 \sqrt{\frac{L}{N_{p} A^{M}}}
$$

\subsubsection{Constraint functions}

There are two groups of constraints that remain to be made explicit.

\section{Kinematics}

Using equations (1) and (35), the kinematic constraints (24) and (25) are obtained as follows:

$$
\begin{aligned}
\frac{10^{3} V_{c} f_{z} Z}{\pi D} & \leq V_{f}^{M} \\
\sqrt{J^{M} \frac{10^{3} V_{c} f_{z} Z}{\pi D}} & \leq A^{M}
\end{aligned}
$$

\section{Cutting forces}

There are several mechanistic models for cutting forces in the literature, as for example [4, 5, 23]. All of these models are relevant to our optimization problem. For validating our methodology, we use the mechanistic model of [4], which is applied on the material Al356 alloy, plunge milled with a Sandvik tool referenced as R210-025A20-09M. This model gives the analytical formulas of cutting forces to be used in constraints (26), (27), and (28). Based on this model, we propose the following expressions of the maximal cutting forces: 


$$
\begin{aligned}
& F_{t}^{\max }\left(V_{c}, f_{z}, N_{p}\right)=F_{t}^{\max }\left(f_{z}, N_{p}\right)=325.17\left(\cos \left(10^{\circ}\right) f_{z}\right)^{-0.418} \frac{L}{N_{p}} f_{z} \\
& F_{r}^{\max }\left(V_{c}, f_{z}, N_{p}\right)=F_{r}^{\max }\left(f_{z}, N_{p}\right)=202.85\left(\cos \left(10^{\circ}\right) f_{z}\right)^{-0.418} \frac{L}{N_{p}} f_{z} \\
& F_{a}^{\max }\left(V_{c}, f_{z}, N_{p}\right)=F_{a}^{\max }\left(f_{z}, N_{p}\right)=130.33\left(\cos \left(10^{\circ}\right) f_{z}\right)^{-0.682} \frac{L}{N_{p}} f_{z}
\end{aligned}
$$

Remark that these forces do not depend upon $V_{c}$, contrary for example to the model proposed by Danis et al. [5] to plunge milling magnesium alloys. However, this is without loss of generality for our optimization methodology.

Finally, the maximal machining power constraint (29) is straightforwardly obtained:

$$
325.17\left(\cos \left(10^{\circ}\right) f_{z}\right)^{-0.418} \frac{L}{N_{p}} f_{z} V_{c} \leqslant P^{M}
$$

\subsection{Numerical results}

All machining times reported throughout this section correspond to simulated machining times, computed following equations (30), (34), (39) and (40). In order to ensure consistency with real machining times, we perform some actual tests (physical experiments) on the CNC machine tool DMU85 Monoblock with a Siemens $840 \mathrm{D}$ CNC controller. We observe that the difference between simulated and real machining times always remains below $6 \%$. As it will be seen later, this is much lower than the gains obtained with our optimization methodology. This thereby validates the physical models on which our optimization formulation relies. Numerical tests are carried out using the same material, A356 alloy, and the same plunge milling tool (Sandvik R210-Q25A20-09 M) as in [4].

The optimization problem we presented belongs to a class of problems (NP hard) known to be very difficult to solve, as it is nonlinear and involves both continuous and integer variables. The optimization model we propose is implemented in AMPL modeling language [8], and the optimization problem is solved using the state-of-the-art solver COUENNE [2] for Mixed-Integer Nonlinear Problems (MINLP). The default setting is used. In order to be able to use such an efficient global optimization solver, we replace equations (41), (42), and (43) with a rational-function model that fits very accurately these equations on the relevant definition domain. 
Tests with the above optimization software were performed on a $2.66 \mathrm{GHz}$ Intel Xeon (octo core) processor with 32 GB of RAM and Linux Operating System. To give a rough idea, one complete run of the optimization (on one test problem) involves between 0.23 and 0.56 seconds of CPU time. Recall that one run involves one elementary trajectory. Therefore, a complete manufacturing of one workpiece will require to sum up this computing time over all elementary trajectories.

We present below eight test cases, each of which is followed by the results obtained after optimization. These tests are performed varying tool characteristics, the machine power and kinematics, the cutting-force constraints as well as the workpiece characteristics (the main variations are emphasized in bold characters in the tables below). These tests give an overview of real cases that can be met in practice on milled engine housings. In all tests, we keep constant: the tool diameter $(D)$, the length of the elementary trajectory $(L)$, the maximum machine power upper bound $\left(P^{M}\right)$ and the lower and upper bounds of the decision variables. These values are displayed in Table 1. The maximum machine power is set at a large value so as to improve the range of possible values for the cutting parameters. The optimization results are compared with a standard industrial setting (SIS), based on engineering know-how in such a way to satisfy the constraints on cutting parameters, kinematics and cutting forces, while ensuring good machining time performance. More precisely, the following procedure is applied. The values of $V_{c}, f_{z}$, and $a_{e}$ are chosen as high as possible so as to fulfill the constraints. The three values are defined sequentially by using a trial-and-error approach. First, $a_{e}$ is taken close to the upper bound $a_{e}^{M}$, then the value of $f_{z}$ is deduced from the cutting force model, and finally, the value of $V_{c}$ follows from the other constraints. The number of plunges, $N_{p}$, is simply obtained by rounding above the ratio $L / a_{e}$. This is in contrast with the MINLP approach which computes optimal values for $V_{c}, f_{z}, N_{p}$, where $N_{p}$ is constrained to be integer. The value of $a_{e}$ is then simply deduced from (6).

We use the above-mentioned DMU85 machine and two other (more performing) machines, presented in [16].

For the sake of simplicity, among the three cutting-force constraints (26)(28), only the first one (equation (26)) is taken into account (and we observed a posteriori that the two other constraints are not violated at the solution found).

We next present the results for the eight test cases. For each approach (SIS and MINLP) we report: the values of the variables, $V_{c}, f_{z}, N_{p}$ and $a_{e}$; 
Table 1: Values of the parameters that are kept constant throughout the tests

\begin{tabular}{c|ccccc}
\hline $\begin{array}{c}\text { parameter } \\
\text { units }\end{array}$ & $\begin{array}{c}L \\
\mathrm{~mm}\end{array}$ & $\begin{array}{c}L \\
\mathrm{~mm}\end{array}$ & $\begin{array}{c}P^{M} \\
\mathrm{~kW}\end{array}$ & $\begin{array}{c}V_{c}^{m} \\
\mathrm{~m} / \mathrm{min}\end{array}$ & $\begin{array}{c}V_{c}^{M} \\
\mathrm{~m} / \mathrm{min}\end{array}$ \\
\hline value & 25 & 200 & 20 & 200 & 1250 \\
\hline \hline parameter & $f_{z}^{m}$ & $f_{z}^{M}$ & $a_{e}^{m}$ & $a_{e}^{M}$ & \\
units & $\mathrm{mm} / \mathrm{rev} /$ tooth & $\mathrm{mm} / \mathrm{rev} /$ tooth & $\mathrm{mm}$ & $\mathrm{mm}$ & \\
\hline value & 0.05 & 1 & 0.5 & 8 & \\
\hline
\end{tabular}

the three plunging-phase times, $t_{p}, t_{r}$ and $t_{o}$; the total machining time, $T$, for one elementary trajectory. Finally the last column of the tables reports the relative gain of MINLP over SIS total machining time.

The first test (Table 2) considers the standard CNC machine tool DMU85 Monoblock with a high value of $F_{t}^{M}$ so that the tool load is not much constrained.

Table 2: Test 1: input data

\begin{tabular}{c|cccccc}
\hline $\begin{array}{c}\text { parameter } \\
\text { units }\end{array}$ & $\begin{array}{c}L_{p} \\
\mathrm{~mm}\end{array}$ & $\begin{array}{c}A^{M} \\
\mathrm{~m} / \mathrm{s}^{2}\end{array}$ & $\begin{array}{c}J^{M} \\
\mathrm{~m} / \mathrm{s}^{3}\end{array}$ & $\begin{array}{c}V_{R}^{M} \\
\mathrm{~m} / \mathrm{min}\end{array}$ & $\begin{array}{c}V_{f}^{M} \\
\mathrm{~m} / \mathrm{min}\end{array}$ & $\begin{array}{c}F_{t}^{M} \\
\mathrm{~N}\end{array}$ \\
\hline value & 75 & 6 & 40 & 40 & 40 & 900 \\
\hline
\end{tabular}

Table 3 displays the results obtained after optimization compared with the standard industrial setting (SIS) procedure described above. One observes that for one given elementary trajectory, the optimization results are better, but close to the one obtained by SIS. Note that the SIS solution provides a non-integer value of $L / a_{e}=26.67$ which was then rounded up to 27 in order to obtain a feasible number of plunges.

The second test (Table 4) considers the same machine as for Test 1, but Test 2 involves a lower value of $F_{t}^{M}$ so as to reduce the bending and/or the vibrations of the tool. The input value for $F_{t}^{M}$ is chosen taking into account the relation between the deflection of the tool, the cutting forces and experience of practitioners. 
Table 3: Test 1: optimization results

\begin{tabular}{c|cccc|ccc|cc}
\hline & $V_{c}$ & $f_{z}$ & $N_{p}$ & $a_{e}$ & $t_{p}$ & $t_{r}$ & $t_{o}$ & $T$ & gain \\
units & $\mathrm{m} / \mathrm{min}$ & $\mathrm{mm} / \mathrm{rev} /$ tooth & & $\mathrm{mm}$ & $\mathrm{s}$ & $\mathrm{s}$ & $\mathrm{s}$ & $\mathrm{s}$ & $\%$ \\
\hline SIS & 1250 & 0.194 & 27 & 7.50 & 0.85 & 0.22 & 0.071 & 30.80 & \\
\hline MINLP & 1250 & 0.198 & 27 & 7.41 & 0.83 & 0.22 & 0.070 & 30.45 & 1.1 \\
\hline
\end{tabular}

Table 4: Test 2: input data

\begin{tabular}{c|cccccc}
\hline parameter & $L_{p}$ & $A^{M}$ & $J^{M}$ & $V_{R}^{M}$ & $V_{f}^{M}$ & $F_{t}^{M}$ \\
\hline value & 75 & 6 & 40 & 40 & 40 & $\mathbf{6 0 0}$ \\
\hline
\end{tabular}

The MINLP solution of Table 5 increases the number of plunges with respect to the SIS solution (going from 27 to 39) and thereby decreases the radial offset $\left(a_{e}\right)$ from 7.5 down to 5.13. Moreover, the feed per tooth, $f_{z}$, increases from 0.087 to 0.182 and the cutting speed, $V_{c}$, remains constant. Thus, the optimized process allows one to perform more plunges at a higher speed. The gain in terms of machining time is above $15 \%$. Here, one already observes the benefit of the optimization which yields an interesting yet unpredictable result that is substantially different from the engineering know-how SIS methodology.

Table 5: Test 2: optimization results

\begin{tabular}{c|cccc|ccc|cc}
\hline & $V_{c}$ & $f_{z}$ & $N_{p}$ & $a_{e}$ & $t_{p}$ & $t_{r}$ & $t_{o}$ & $T$ & gain \\
\hline SIS & 1250 & 0.087 & 27 & 7.50 & 1.70 & 0.22 & 0.071 & 53.96 & \\
\hline MINLP & 1250 & 0.182 & 39 & 5.13 & 0.89 & 0.22 & 0.058 & 45.69 & 15.3 \\
\hline
\end{tabular}

Test 3 (Table 6) involves a higher workpiece (higher value of $L_{p}$ ), all other conditions remaining as for Test 2.

Table 6: Test 3: input data

\begin{tabular}{c|cccccc}
\hline parameter & $L_{p}$ & $A^{M}$ & $J^{M}$ & $V_{R}^{M}$ & $V_{f}^{M}$ & $F_{t}^{M}$ \\
\hline value & $\mathbf{1 0 0}$ & 6 & 40 & 40 & 40 & 600 \\
\hline
\end{tabular}

The optimization results of Table 7 show that the gain in machining time grows further with a higher workpiece. 
Table 7: Test 3: optimization results

\begin{tabular}{c|cccc|ccc|cc}
\hline & $V_{c}$ & $f_{z}$ & $N_{p}$ & $a_{e}$ & $t_{p}$ & $t_{r}$ & $t_{o}$ & $T$ & gain \\
\hline SIS & 1250 & 0.087 & 27 & 7.50 & 2.25 & 0.26 & 0.071 & 69.52 & \\
\hline MINLP & 1250 & 0.197 & 41 & 4.88 & 1.07 & 0.26 & 0.057 & 57.02 & 18.0 \\
\hline
\end{tabular}

Let us now consider, in Test 4 (Table 8), an even higher workpiece. The length of the tool being increased, its stiffness thereby reduces and, as a consequence, one must set a stricter cutting-force constraint (lower $F_{t}^{M}$ ) in order to avoid bending and/or vibration of the tool.

Table 8: Test 4: input data

\begin{tabular}{c|cccccc}
\hline parameter & $L_{p}$ & $A^{M}$ & $J^{M}$ & $V_{R}^{M}$ & $V_{f}^{M}$ & $F_{t}^{M}$ \\
\hline value & $\mathbf{1 2 5}$ & 6 & 40 & 40 & 40 & $\mathbf{5 0 0}$ \\
\hline
\end{tabular}

As shown in Table 9, the gain obtained by the MINLP solution (over SIS) for Test 4 is substantial: the optimized process yields a very large number of plunges ( $N_{p}=91$, as opposed to 27 for SIS) at a very high programmed feedrate, $V_{f}=31.826 \mathrm{~m} / \mathrm{min}$ (from equation 1 ). The gain in terms of machining time is $41.8 \%$.

Table 9: Test 4: optimization results

\begin{tabular}{c|cccc|ccc|cc}
\hline & $V_{c}$ & $f_{z}$ & $N_{p}$ & $a_{e}$ & $t_{p}$ & $t_{r}$ & $t_{o}$ & $T$ & gain \\
\hline SIS & 1250 & 0.054 & 27 & 7.50 & 4.43 & 0.29 & 0.071 & 129.21 & \\
\hline MINLP & 1250 & 1.00 & 91 & 2.20 & 0.50 & 0.29 & 0.038 & 75.20 & 41.8 \\
\hline
\end{tabular}

Test 5 (Table 10) involves a high-performance machine, featuring a higher (compared with the previous tests) maximum axis acceleration reachable, $A^{M}$. The other parameter values are the same as those of Test 2 .

The optimization results (Table 11) show, when compared with those of Test 2, an even higher gain (17.2\% as compared with $15.3 \%)$.

Test 6 (Table 12) differs from Test 5 only in the value of the maximum axis jerk reachable, $J^{M}$, which is much increased. The other parameter values are the same as those of Test 2. 
Table 10: Test 5: input data

\begin{tabular}{c|cccccc}
\hline parameter & $L_{p}$ & $A^{M}$ & $J^{M}$ & $V_{R}^{M}$ & $V_{f}^{M}$ & $F_{t}^{M}$ \\
\hline value & 75 & $\mathbf{1 0}$ & 40 & 40 & 40 & 600 \\
\hline
\end{tabular}

Table 11: Test 5: optimization results

\begin{tabular}{c|cccc|ccc|cc}
\hline & $V_{c}$ & $f_{z}$ & $N_{p}$ & $a_{e}$ & $t_{p}$ & $t_{r}$ & $t_{o}$ & $T$ & gain \\
\hline SIS & 1250 & 0.087 & 27 & 7.50 & 1.70 & 0.17 & 0.055 & 52.17 & \\
\hline MINLP & 1250 & 0.190 & 40 & 5.00 & 0.86 & 0.17 & 0.045 & 43.17 & 17.2 \\
\hline
\end{tabular}

Table 12: Test 6: input data

\begin{tabular}{c|cccccc}
\hline parameter & $L_{p}$ & $A^{M}$ & $J^{M}$ & $V_{R}^{M}$ & $V_{f}^{M}$ & $F_{t}^{M}$ \\
\hline value & 75 & 10 & $\mathbf{1 0 0}$ & 40 & 40 & 600 \\
\hline
\end{tabular}

Table 13 reveals a further improvement in machining time, $T$. To summarize, when considering only the MINLP solutions of Tests 2, 5 and 6, one remarks that increasing either $A^{M}$ or $J^{M}$ yields gains in machining times, more plunges and higher feedrates.

Table 13: Test 6: optimization results

\begin{tabular}{c|cccc|ccc|cc}
\hline & $V_{c}$ & $f_{z}$ & $N_{p}$ & $a_{e}$ & $t_{p}$ & $t_{r}$ & $t_{o}$ & $T$ & gain \\
\hline SIS & 1250 & 0.087 & 27 & 7.50 & 1.68 & 0.17 & 0.055 & 51.39 & \\
\hline MINLP & 1250 & 0.212 & 43 & 4.65 & 0.75 & 0.17 & 0.043 & 41.37 & 19.5 \\
\hline
\end{tabular}

Recall that from Test 3 to Test 4 , we increased $L_{p}$ and therefore decreased $F_{t}^{M}$ to take stiffness into account. Test 7 (Table 14) comes back to the main settings of Test 4 together with the high-performance machine of Tests 5 and 6 .

As shown on Table 15, the gain with respect to the engineering know-how SIS methodology is striking: $52.7 \%$. When comparing the MINLP solutions obtained for Tests 4 and 7 (that differs with respect to the performance of the machine), the gain goes from $41.8 \%$ to $52.7 \%$. Thus, higher workpieces (and corresponding tool-stiffness constraints) conduct to better results. 
Table 14: Test 7: input data

\begin{tabular}{c|cccccc}
\hline parameter & $L_{p}$ & $A^{M}$ & $J^{M}$ & $V_{R}^{M}$ & $V_{f}^{M}$ & $F_{t}^{M}$ \\
\hline value & $\mathbf{1 2 5}$ & 10 & 100 & 40 & 40 & $\mathbf{5 0 0}$ \\
\hline
\end{tabular}

Table 15: Test 7: optimization results

\begin{tabular}{c|cccc|ccc|cc}
\hline & $V_{c}$ & $f_{z}$ & $N_{p}$ & $a_{e}$ & $t_{p}$ & $t_{r}$ & $t_{o}$ & $T$ & gain \\
\hline SIS & 1250 & 0.054 & 27 & 7.50 & 4.40 & 0.22 & 0.055 & 126.41 & \\
\hline MINLP & 1250 & 1.00 & 91 & 2.20 & 0.40 & 0.22 & 0.030 & 59.79 & 52.7 \\
\hline
\end{tabular}

Test 8 (Table 16) considers an even more performing machine tool: we use the characteristics of a parallel kinematic machine presented in [16] for plunge milling. Its maximum acceleration value is $A^{M}=15 \mathrm{~m} \cdot \mathrm{s}^{-2}$ and $V_{f}^{M}=50$ $\mathrm{m} / \mathrm{min}$.

Table 16: Test 8: input data

\begin{tabular}{c|cccccc}
\hline parameter & $L_{p}$ & $A^{M}$ & $J^{M}$ & $V_{R}^{M}$ & $V_{f}^{M}$ & $F_{t}^{M}$ \\
\hline value & 125 & $\mathbf{1 5}$ & 100 & $\mathbf{5 0}$ & $\mathbf{5 0}$ & 500 \\
\hline
\end{tabular}

Table 17 displays a gain that continues to grow: $55.6 \%$. When comparing the optimization solutions obtained for Tests 4, 7 and 8 (in order of increasing performance of the machine tool used), the gains are $41.8 \%, 52.7 \%$ and $55.6 \%$.

Table 17: Test 8: optimization results

\begin{tabular}{c|cccc|ccc|cc}
\hline & $V_{c}$ & $f_{z}$ & $N_{p}$ & $a_{e}$ & $t_{p}$ & $t_{r}$ & $t_{o}$ & $T$ & gain \\
\hline SIS & 1250 & 0.054 & 27 & 7.50 & 4.40 & 0.18 & 0.044 & 125.03 & \\
\hline MINLP & 1250 & 1.00 & 91 & 2.20 & 0.40 & 0.18 & 0.024 & 55.56 & 55.6 \\
\hline
\end{tabular}

\section{Conclusions}

We propose a mathematical formulation of the cutting-parameter optimization in plunge milling, taking into account the lengths of the tool trajecto- 
ries, control laws and cutting forces. This formulation takes the form of an MINLP problem whose optimization variables include the (integer) number of plunges.

The advantages of our approach over traditional machining optimization is twofold. First, our method provides a specific set of cutting parameters tailored to each elementary trajectory, which, together, constitute the tool trajectory. Second, for each elementary trajectory, our formulation yields equidistant plunges that are optimal with respect to all the cutting parameters. This is in contrast with standard machining optimization that uses only continuous variables. Indeed, such standard approaches not only consider a same set of cutting parameters over the whole trajectory, but moreover adjust a posteriori the number of plunges, while keeping fixed the values of the other cutting parameters, which is necessarily suboptimal. This loss of efficiency is then summed up over all the elementary trajectories.

We present and discuss computational results, obtained through the use of modern general-purpose MINLP software, validated on CNC machines. The tests proposed cover a wide range of input parameter values (workpiece and tool characteristics, machine performances, and tool and machine constraints). Our results emphasize the fact that optimal values of the cutting parameters are very sensitive to the various constraints (cutting-force constraints and maximal machining power) and are difficult to predict by standard approaches. The more performing machine one uses, the better results our optimization methodology yields. Gains as high as 55\% for highperformance machine tools are obtained when compared with standard industrial setting based on engineering know-how.

Future research will extend this work by optimizing moreover both the tool choice and the associated tool-path trajectory. Moreover, one could focus on attempting to search for cutting-parameter values that are robust with respect to uncertainty in input data.

\section{A Impact of control laws on machining time}

In this appendix, we show the strong impact of control laws on machining time, especially when high speeds are used. This is illustrated in different test cases by comparing real machining time and simulated machining time provided by CAM software that does not take into account the control laws.

For illustrating our purpose, we have performed plunge milling tests on a 
DMU50 evolution 5-axis NC machine (with a Siemens 840D CNC controller), for which the machining times have been recorded. In commercial CAM software, the kinematic machine-tool characteristics can be considered, but generally the control law profiles cannot be taken into account. To compare real machining times with simulation times, two CAM software (CATIA V5 and Delcam) have been used. The tests have been performed (Table 18) on different workpieces with the following kinematic characteristics: for $i \in$ $\{x, y, z\}, V_{R i}^{M}=V_{R}^{M}=50 \mathrm{~m} \cdot \mathrm{min}^{-1}, V_{f i}^{M}=V_{f}^{M}=24 \mathrm{~m} \cdot \mathrm{min}^{-1}, J_{i}^{M}=$ $J^{M}=40 \mathrm{~m} \cdot \mathrm{s}^{-3}, A_{i}^{M}=A^{M}=4.9 \mathrm{~m} \cdot \mathrm{s}^{-2}$. For the first workpiece, Catia V5 simulates the machining time equal to $91 \mathrm{~s}$, compared to $235 \mathrm{~s}$ of real time machining. For the second workpiece tested, Delcam has provided a simulation time of $86 \mathrm{~s}$ against $213.6 \mathrm{~s}$ of machining time. In these two examples, CAM software decreases the actual machining time by about $60 \%$. Table 18 presents all the results of the time differences for various workpieces and programmed feedrates. Errors are in the range [49\%, 61\%] for both CAM software. Nevertheless, depending on the machine characteristics $\left(V_{R}^{M}\right.$, $\left.V_{f}^{M}, A^{M}, J^{M}\right)$, these errors vary significantly. These test results show the criticity of the control laws for the computation of simulated machining times. Therefore, velocity profiles provided by control laws will be integrated into our optimization model.

\begin{tabular}{|c|c|c|c|c|}
\cline { 2 - 5 } \multicolumn{1}{c|}{} & Catia V5 (s) & Delcam (s) & Time machining (s) & errors \\
\hline Test1 & 91 & & 235 & $61 \%$ \\
\hline Test2 & & 86 & 213.6 & $60 \%$ \\
\hline Test3 & 95 & & 186 & $49 \%$ \\
\hline Test4 & 87 & & 158 & $45 \%$ \\
\hline Test5 & 82 & & 174.7 & $53 \%$ \\
\hline
\end{tabular}

Table 18: Comparison of machining time simulated by two CAM software and real time machining for different test cases

\section{References}

[1] A. Banerjee, H.-Y. Feng, and E. V. Bordatchev. Process planning for Floor machining of 2D pockets based on a morphed spiral tool path pattern. Computers \&3 Industrial Engineering, 63(4):971-979, 2012. 
[2] P. Belotti, J. Lee, L. Liberti, F. Margot, and A. Wachter. Branching and bounds tightening techniques for non-convex MINLP. Optimization Methods and Software, 24(4-5):597-634, 2009.

[3] S. Calvel and M. Mongeau. Black-box structural optimization of a mechanical component. Computers \&6 Industrial Engineering, 53(3):514530, 2007.

[4] A. Damir, E.-G. Ng, and M. Elbestawi. Force prediction and stability analysis of plunge milling of systems with rigid and flexible workpiece. The International Journal of Advanced Manufacturing Technology, 54(912):853-877, 2010.

[5] I. Danis, F. Monies, P. Lagarrigue, and N. Wojtowicz. Cutting forces and their modelling in plunge milling of magnesium-rare earth alloys. The International Journal of Advanced Manufacturing Technology, 84(9):1801$1820,2016$.

[6] I. Danis, N. Wojtowicz, F. Monies, and P. Lagarrigue. Influence of dry plunge milling conditions on surface integrity of magnesium alloys. International Journal of Mechatronics and Manufacturing Systems, 7(23):141-156, 2014.

[7] T. Finzer. High speed machining (HSC) of sculptured surfaces in die and mold manufacturing. In G. J. Olling, B. K. Choi, and R. B. Jerard, editors, Machining Impossible Shapes, number 18 in IFIP - The International Federation for Information Processing, pages 333-341. Springer, 1999.

[8] R. Fourer and D. M. Gay. The AMPL Book. Duxbury Press, Pacific Grove, 2002.

[9] P. Gilles, F. Monies, and W. Rubio. Optimum orientation of a torus milling cutter: Method to balance the transversal cutting force. International Journal of Machine Tools and Manufacture, 47(15):2263-2272, 2007.

[10] F. Y. Han, D. H. Zhang, M. Luo, and B. H. Wu. Optimal CNC plunge cutter selection and tool path generation for multi-axis roughing freeform surface impeller channel. The International Journal of Advanced Manufacturing Technology, 71(9-12):1801-1810, 2014. 
[11] H. Juan, S. F. Yu, and B. Y. Lee. The optimal cutting-parameter selection of production cost in HSM for SKD61 tool steels. International Journal of Machine Tools and Manufacture, 43(7):679-686, 2003.

[12] J. H. Ko and Y. Altintas. Time domain model of plunge milling operation. International Journal of Machine Tools and Manufacture, 47(9):1351-1361, 2007.

[13] F. Koenigsberger and A. Sabberwal. An investigation into the cutting force pulsations during milling operations. International Journal of $M a-$ chine Tool Design and Research, 1(1):15-33, 1961.

[14] J. Lee and S. Leyffer. Mixed integer nonlinear programming, volume 154. Springer Science \& Business Media, 2011.

[15] K. Moussaoui, F. Monies, M. Mousseigne, P. Gilles, and W. Rubio. Balancing the transverse cutting force during inclined milling and effect on tool wear: application to Ti6al4v. The International Journal of Advanced Manufacturing Technology, 82(9):1859-1880, 2016.

[16] M. Rauch and J.-Y. Hascoet. Selecting a milling strategy with regard to the machine tool capabilities: application to plunge milling. The International Journal of Advanced Manufacturing Technology, 59(1-4):47-54, 2012.

[17] J. X. Ren, C. F. Yao, D. H. Zhang, Y. L. Xue, and Y. S. Liang. Research on tool path planning method of four-axis high-efficiency slot plunge milling for open blisk. The International Journal of Advanced Manufacturing Technology, 45(1-2):101-109, 2009.

[18] H. Schulz. High-speed machining. In A. I. Dashchenko, editor, Manufacturing Technologies for Machines of the Future, pages 197-214. Springer, 2003.

[19] Siemens. Technical Online-Documentation for SINUMERIK, SINAMICS and SIMOTICS - ID: 109476679 - Industry Support Siemens, 2015.

[20] C. Sun, Y. Wang, and N. Huang. A new plunge milling tool path generation method for radial depth control using medial axis transform. The International Journal of Advanced Manufacturing Technology, 76(9):1575-1582, 2015. 
[21] J. Tlusty and P. MacNeil. Dynamics of cutting forces in end milling. Annals CIRP, 24:21-25, 1975.

[22] K. Zhuang, X. Zhang, D. Zhang, and H. Ding. On cutting parameters selection for plunge milling of heat-resistant-super-alloys based on precise cutting geometry. Journal of Materials Processing Technology, 213(8):1378-1386, 2013.

[23] K. Zhuang, X. Zhang, X. Zhang, and H. Ding. Force prediction in plunge milling of inconel 718. In C.-Y. Su, S. Rakheja, and H. Liu, editors, Intelligent Robotics and Applications, number 7507 in Lecture Notes in Computer Science, pages 255-263. Springer Berlin Heidelberg, 2012 . 